\title{
« Faites-vous vacciner vos enfants? »
}

\author{
Kieran M. Moore, MD, MMS; ${ }^{*}$ Marco L.A. Sivilotti, MD, $\mathrm{MSc}^{\dagger}$ \\ Voir L'ARTicle connexe À LA PAge 69 \\ English Version on PAge 14
}

A u moment où nous écrivons ce commentaire, le pays vient d'entrer dans la deuxième semaine de vaccination de masse contre le virus de la grippe pandémique $\mathrm{pH} 1 \mathrm{~N} 1$. Les cyniques suggèrent que le $\ll \mathrm{p} »$ minuscule signifie « pandémonium » plutôt que «pandémique ». Les bienfaits de la vaccination font l'objet de débats dans les postes infirmiers, les arénas de hockey et lors d'une séance d'urgence du Parlement fédéral. Une conséquence involontaire de cette mesure de santé publique est que le scepticisme à l'égard du vaccin a atteint son paroxysme.

Une telle réaction n'est pas vraiment surprenante, surtout si l'on considère la capacité de l'histoire de se répéter. Nombreux sont ceux qui croient que le scepticisme à l'égard du vaccin a pénétré la conscience publique après l'échec de la campagne de vaccination de masse contre une autre éclosion de grippe porcine en 1976. La méfiance envers les décrets du gouvernement a plus tard alimenté les propos de la science de pacotille qui associent la vaccination à l'autisme ${ }^{1-3}$. Lorsque des peurs irrationnelles sont inoculées dans un milieu de culture de désinformation amplifiée par l'Internet dans lequel on ajoute des préoccupations déplacées concernant la toxicité du thimérosal, et que ces peurs sont nourries par des opinions de soi-disant experts qui tirent un profit des cliniques privées de chélation et sont motivés par d'autres intérêts personnels, l'opposition à la vaccination universelle des enfants a un fort potentiel de propagation « virale $»^{4,5}$. Quelles seront, cette année, les conséquences ultimes du programme de vaccination contre la grippe?

Dans ce numéro du JCMU, Grunau et Olson ${ }^{6}$ présentent un cas de tétanos en Colombie-Britannique chez un enfant de 7 ans non vacciné. Ce cas est un rappel émou- vant des dangers de la complaisance face aux réussites en matière de santé publique d'autres maladies devenues rares. Grâce à la vaccination généralisée, l'incidence annuelle du tétanos est tombée à moins de 0,1 cas par million dans le monde industrialisé. Les récentes éditions du manuel de Fleisher et de ses collaborateurs Textbook of Pediatric Emergency Medicine ne mentionnent cette maladie qu'en passant. Peu d'entre nous s'attendent à poser un diagnostic de tétanos, même si, au quotidien, on pose machinalement des questions aux patients sur leur statut vaccinal et que l'on prescrit des doses vaccinales d'anatoxines tétaniques. Quand des programmes de vaccination efficaces réduisent l'incidence d'une maladie comme le tétanos à des niveaux si faibles, certains en viennent naturellement à remettre en question la nécessité de la vaccination. Pourtant, Clostridium tetani demeure une bactérie omniprésente dans l'environnement, et un nombre important de Canadiens de tous âges ont des taux sériques d'antitoxines antitétaniques non protecteurs'. À l'échelle planétaire, faute de vaccination universelle, on estime que le tétanos tue annuellement des centaines de milliers de personnes ${ }^{9}$.

Donc, dans notre analyse actuelle de la campagne $\mathrm{pH} 1 \mathrm{~N} 1$, nous devons nous souvenir des leçons de l'histoire. Nous devons également chercher à fournir des avis scientifiques rationnels et des preuves et à disposer d'une bonne dose de bon sens; ce sont là les principales garanties contre la panique et la désinformation. Les médecins d'urgence ont des occasions en or de renforcer les interventions de santé publique : abandon du tabagisme, port du casque de moto, promotion de sièges d'auto pour nourrissons et consommation responsable d'alcool, par exemple. L'incertitude scientifique et la divergence d'opinions peuvent entraîner des conflits,

Des *départements de médecine familiale et de médecine d'urgence, Université Queen's, Kingston (Ontario), et des †départements de médecine d'urgence, de pharmacologie et de toxicologie, Université Queen's, Kingston (Ontario)

Les opinions exprimées dans les éditoriaux sont celles des auteurs; elles ne reflètent pas nécessairement celles du JCMU ou de l'Association canadienne des médecins d'urgence. 
mais les valeurs sociétales libérales peuvent l'emporter sur les choix individuels, surtout lorsque le parent fait un choix au nom d'un mineur. On n'hésite pas à dire qu'il est absurde de conduire une automobile avec un enfant non attaché sur le siège avant parce que le parent croit qu'exposer l'enfant à des traumatismes crâniens sublétaux va augmenter l'épaisseur de son cerveau et ainsi sa résistance à d'autres traumatismes. Alors, pourquoi sommes-nous moins portés à exprimer notre opposition aux théories voulant que la vaccination affaiblisse le système immunitaire ou empoisonne le système nerveux?

La vaccination rapide et généralisée contre la grippe est le moyen le plus efficace de réduire l'incidence de la grippe sur nous-mêmes et la communauté. Seulement entre un quart et la moitié des travailleurs de la santé dans les hôpitaux et les établissements de soins de longue durée reçoivent le vaccin contre la grippe saisonnière, malgré les preuves de son effet positif sur la mortalité de leurs patients ${ }^{10,11}$. Pendant que le débat sur la vaccination contre la grippe $\mathrm{pH} 1 \mathrm{~N} 1$ se poursuit, nous devons nous rappeler que certains vont extrapoler ce scepticisme à d'autres maladies et à d'autres vaccins. Alors, combien de fois ce rapport de cas de tétanos évitable se répétera-t-il?

La prévention est presque toujours préférable à n'importe quel traitement. Nous devons exprimer notre soutien cohérent et non équivoque des mesures de santé préventives qui comportent des avantages importants et prouvés. Dans le sillage de la pandémie de 2009, nous ne devons pas oublier les avantages que procure la vaccination pour les individus et la population dans son ensemble. La vue d'un enfant fébrile et sous-vacciné est un moment propice à l'enseignement et une occasion de dissiper les mythes entourant la vaccination et ce, sans tenir compte des autres leçons que le pH1N1 nous a apprises l'automne dernier.

Conflits d'intérêts : aucun déclaré.
Mots clés : tétanos, grippe humaine, vaccination, immunisation de masse

\section{RÉFÉRENCES}

1. DeStefano F. Vaccines and autism: evidence does not support a causal association. Clin Pharmacol Ther 2007;82:756-9.

2. Baker JP. Mercury, vaccines, and autism: one controversy, three histories. Am 7 Public Health 2008;98:244-53.

3. Stewart AM. When vaccine injury claims go to court. $N$ Engl J Med 2009;360:2498-500.

4. Offit PA. Thimerosal and vaccines - a cautionary tale. N Engl J Med 2007;357:1278-9.

5. Thimérosal : nouvelle déclaration. Une déclaration d'un comité consultatif (DCC). Relevé des maladies transmissibles au Canada [1188-4169]. Ottawa (Ontario) : Comité consultatif national de l'immunisation; 2007;33(DCC-6):1-13.

6. Grunau BE, Olson J. An interesting presentation of pediatric tetanus. CJEM 12;1:69-72.

7. Fleisher GR, Ludwig S, Henretig FM, editors. Textbook of pediatric emergency medicine. 5th ed. Philadelphia (PA): Lippincott Williams \& Wilkins; 2006.

8. Guide canadien d'immunisation. $7^{\mathrm{e}}$ édition. Ottawa (Ontario) : Agence de la santé publique du Canada; 2006.

9. Relevé épidémiologique hebdomadaire. Genève $(\mathrm{CH})$ : Organisation mondiale de la Santé; 2006. 197-208.

10. Comité consultatif national de l'immunisation. Déclaration sur la vaccination antigrippale pour la saison 2004-2005. Relevé des maladies transmissibles au Canada 2004;30(DCC-3): $1-32$.

11. Comité consultatif national de l'immunisation. Déclaration sur la vaccination antigrippale pour la saison 2008-2009. Relevé des maladies transmissibles au Canada 2008;34(DCC-3): $1-46$.

Correspondance : $\mathrm{D}^{\mathrm{r}}$ Marco Sivilotti, Département de médecine d'urgence, 76, rue Stuart, Kingston (Ontario) K7L 2V7; fax : 613 5481374; marco.sivilotti@queensu.ca 